Proceedings

\title{
A Novel Intensification Strategy for Wet Media Milling of Drug Suspensions: Bead Mixtures ${ }^{\dagger}$
}

\author{
Gulenay Guner, Manisha Kannan, Matthew Berrios, Nathaniel Parker and Ecevit Bilgili * \\ Otto H. York Department of Chemical and Materials Engineering New Jersey Institute of Technology, \\ Newark, NJ, USA; gg357@njit.edu (G.G.); mk835@njit.edu (M.K); mb756@njit.edu (M.B.); ndp46@njit.edu \\ (N.P.) \\ * Correspondence: bilgece@njit.edu; Tel.: +1-973-596-2998 \\ + Presented at the 1st International Electronic Conference on Pharmaceutics, 1-15 December 2020; Available \\ online: https://iecp2020.sciforum.net/.
}

Received: date; Accepted: date; Published: date

\begin{abstract}
Nano-sized drug particle formation is one of the most popular approaches in dissolution enhancement of poorly water-soluble drugs. However, it requires process optimization since it is a time consuming and costly method. The aim of this study is to investigate the impact of various mixtures of crosslinked polystyrene (CPS) and yttrium-stabilized zirconia (YSZ) beads (YSZ:CPS = 0:100-100:0 \%v:\%v) on the breakage kinetics and energy consumption during the wet stirred media milling (WSMM) of fenofibrate (FNB, BCS II drug). Five WSMM experiments were conducted using $3000 \mathrm{rpm}$ stirrer speed and 50\% volumetric bead loading with a suspension of $10 \%$ FNB, $7.5 \%$ HPC$\mathrm{L}$, and $0.05 \%$ SDS. Laser diffraction was used for the determination of particle sizes. Breakage rate, power required by the mill, and specific energy consumption were analyzed for all mixture runs. The experimental data show that YSZ beads provided faster breakage than the CPS beads, as signified by the lower time constant of the former. On the other hand, CPS beads required lower stirrer power than the YSZ beads. When both cycle time and specific energy consumption were considered for process optimality, YSZ-CPS bead mixtures performed better than YSZ or CPS alone and the optimal bead mixture was found.
\end{abstract}

Keywords: poorly water-soluble drugs; nanoparticles; wet stirred media milling; bead mixtures; process optimization

\section{Introduction}

The number of poorly water-soluble drug candidates coming out of drug discovery has increased tremendously over the past few decades. Their formulation into efficacious dosage forms presents various challenges [1]. Nano-sized drug particle formation is one of the most popular approaches in dissolution enhancement of poorly water-soluble drugs [2]. Nanoparticles in pharmaceutical studies are considered as crystalline particles that have a size in the $50-500 \mathrm{~nm}$ range. Due to the high surface area of nanoparticles compared to their micron-sized counterparts, a higher dissolution rate is observed for the drug nanoparticles. Besides the higher mass transfer coefficient associated with drug nanoparticles, sub- $100 \mathrm{~nm}$ particles exhibit a saturation solubility owing to their high curvature higher than the thermodynamic solubility [3]. These advantages led to the use of nanosuspensions or nanocomposites in several marketed products that has various delivery routes such as oral, parental, pulmonary, ocular, and dermal [4-6].

Wet stirred media milling (WSMM) has been the most preferred method for producing drug nanoususpensions used in the manufacturing of these marketed products and in scientific studies according to the recent literature review [7]. The reason for this high demand in WSMM is that it has 
the potential of producing high drug-loaded, stable nanosuspensions and it is a robust, reproducible, scalable, solvent-free, and environmentally benign process [8-10].

A schematic of the WSMM process operating in the recirculation mode is illustrated in Figure 1. During WSMM, a pre-suspension, which contains drug and an aqueous solution of stabilizers i.e., polymers and surfactants, is circulated between the holding tank and the milling chamber where the beads are held inside by the help of a screen. As the slurry is stirred by the mill, turbulent flow emerges causing frequent bead-bead collisions that break the drug particles captured and compressed between the beads [8].

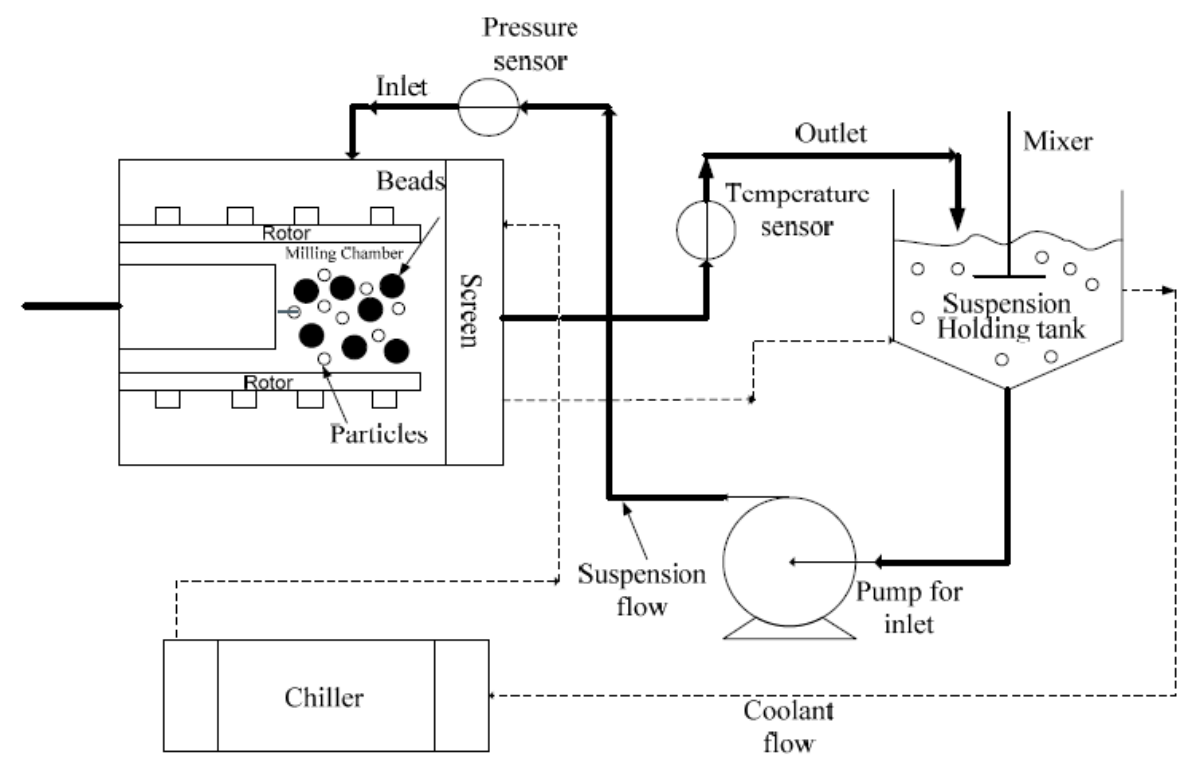

Figure 1. Schematic of wet stirred medial mill in recirculation mode of operation.

Most of the studies in WSMM focus on the formulation aspects such as the aggregation and crystal growth caused by Ostwald ripening and stabilization of drug nanosuspensions $($ see $[7,8]$ and references cited therein). However, there is limited information available for solving the processingoperational challenges such as cost due to high energy consumption, long operating hours, and contamination of drug particles by the beads $[8,11]$. Solving these problems entails a mechanistic understanding of the impact of process parameters such as the stirrer speed, the size and material of the beads, the bead loading, the suspension flow rate, and the drug loading on the breakage kinetics, specific energy consumption, media wear, and milling time required for the desired product fineness $[12,13]$.

Recent efforts in the pharmaceutical WSMM literature have focused on processing challenges and process optimization. Li et al. [14] studied the impact of different sizes of yttrium stabilized zirconia (YSZ) beads on the breakage kinetics of griseofulvin and used a microhydrodynamic model to explain the results. It was found that there is an optimum bead size in each stirrer speed. In light of this study, sub-100 nm drug particles were reached when intensified process conditions were applied by selecting the smallest YSZ beads, highest stirrer speed and flow rate [10]. While these studies were conducted by using only YSZ beads, Parker et al. [15] used crosslinked polystyrene (CPS) and YSZ beads, which are the most commonly used beads in the literature, under different stirrer speed and bead loading conditions where head-to-head comparison of the bead materials was possible. According to the microhydrodynamic analysis, two counteracting advantages of these two bead materials were found: while YSZ beads provided a more frequent compression of drug particles, CPS beads allowed a larger contact area. Overall, YSZ beads provided faster breakage although the energy consumption was also higher but a possible synergistic effect of mixing CPS and YSZ beads on breakage kinetics or energy consumption was not studied. 
The aim of this study is to investigate the impact of mixtures of CPS and YSZ beads on the breakage kinetics and energy consumption at a fixed bead loading and stirrer speed. Fenofibrate (FNB) was selected as a poorly water-soluble model. Five milling experiments were conducted to examine the impact of various volumetric ratios of CPS to YSZ beads $(0: 100-100: 0 \% \mathrm{v}: \% \mathrm{v})$ at 3000 rpm stirrer speed and 0.5 bead loading which are the commonly used conditions in the literature $[16,17]$. Particle sizes of the suspension samples taken at various milling times were measured via laser diffraction and breakage kinetics was studied. In order to characterize the breakage kinetics, three different time constants were defined and analyzed. One of the characteristic times and the specific energy consumption were used to calculate a merit score to assess the overall effects of the use different YSZ:CPS bead mixtures and identify any optimal bead mixture.

\section{Experiments}

\subsection{Materials}

Fenofibrate (FNB, BP grade), which is a BCS Class II drug, was purchased from Jai Radhe Sales (Ahmedabad, India). The aqueous solubility of FNB is $0.8 \mathrm{mg} / \mathrm{L}$ at room temperature [18]. Two stabilizers were used: a non-ionic cellulosic polymer, hydroxypropyl cellulose (HPC, L grade, Nisso America Inc., New York, NY, USA), and an anionic surfactant, sodium dodecyl sulfate (SDS, ACS grade, GFS chemicals, Columbus, OH, USA). Zirmil Y grade YSZ beads and HCC grade CPS beads with a nominal size of $400 \mu \mathrm{m}$ were purchased from Saint Gobain ZirPro (Mountainside, NJ, USA) and Norstone Inc. (Bridgeport, PA, USA) respectively. While YSZ beads have a density of $6000 \mathrm{~kg} / \mathrm{m}^{3}$, the density of CPS beads is $1040 \mathrm{~kg} / \mathrm{m}^{3}$. The actual median sizes of CPS and YSZ beads were measured as $444 \mu \mathrm{m}$ and $405 \mu \mathrm{m}$ respectively via laser diffraction particle size analyzer (Helos/Rodos, Sympatec, NJ, USA) in dry dispersion mode. All suspensions were prepared with deionized water.

\subsection{Wet Stirred Media Milling}

The stabilizer types and their concentrations were selected based on the detailed stability studies performed by our group on FNB [19-21]. The formulation had been selected in such a way that (i) the suspension was stable during and after the milling, and the viscosity of the suspension was high enough to allow for the measurement of the energy consumption accurately while not causing any processability issues such as significant viscous dampening. About $235 \mathrm{~g}$ pre-suspensions that have $10 \% \mathrm{FNB}, 7.5 \% \mathrm{HPC}-\mathrm{L}$, and $0.05 \% \mathrm{SDS}$ (with respect to $200 \mathrm{~g}$ DI water) were prepared under constant shear mixing for $2 \mathrm{~h}$ at $300 \mathrm{rpm}$ (Cat\#. 14-503, Fisher Scientific, Pittsburgh, PA, USA) and were kept under $8{ }^{\circ} \mathrm{C}$ overnight. After that, the pre-suspensions were milled by a Microcer wet stirred media mill (Netzsch Fine Particle Size Technology, LLC, Exton, PA, USA) for $180 \mathrm{~min}$. The volumetric ratio of CPS and YSZ bead mixtures were varied between 100:0-0:100. Suspensions were recirculated between the holding tank and the milling chamber at a volumetric flow rate $Q$ of $126 \mathrm{~mL} / \mathrm{min}$, using a peristaltic pump. The samples were taken from the outlet of the mill at certain time intervals $(2 \mathrm{~s}, \mathrm{~s}$ $=0,1,2, \ldots 7$ ) with the addition of $24 \mathrm{~min}, 48 \mathrm{~min}, 96 \mathrm{~min}$, and $180 \mathrm{~min}$ sampling times. Also, samples were taken at $20 \mathrm{~s}$ and $40 \mathrm{~s}$ since the breakage of the suspension was very fast and earlier data points were needed to discriminate the breakage kinetics of different runs. The final sample was taken from the holding tank and all samples were characterized via laser diffraction. The average power consumption $P$ during the milling of the suspension was determined by dividing the cumulative energy consumption read from the control panel of the mill by the milling time. $P_{\mathrm{w}}$, the average stirrer power per unit volume, was calculated for each run via $P_{\mathrm{w}}=P / V_{\mathrm{m}}$. At the end of the experiments with bead mixtures, the beads were separated by using a salt buffer due to their significant density differences. The sank YSZ beads were collected from the bottom of a separation funnel and the separated beads were cleaned and dried to be reused.

\subsection{Particle Sizing via Laser Diffraction}

Particle size distribution (PSD) of the FNB suspensions at various milling times was determined by laser diffraction using LS 13-320 Beckman Coulter instrument (Brea, CA, USA). In all 
measurements, the polarized intensity differential scattering (PIDS) was maintained between $40 \%$ and $50 \%$ while the obscuration was maintained below $8 \%$. PSD was computed by the software using the Mie scattering theory. Refractive indices of 1.55 and 1.33 were used for FNB and water (measurement medium), respectively. Before each measurement, a $\sim 1.0 \mathrm{~mL}$ suspension sample was dispersed into $5.0 \mathrm{~mL}$ stabilizer solution using a vortex mixer (Fisher Scientific Digital Vortex Mixer, Model No: 945415, Pittsburgh, PA, USA) at $1500 \mathrm{rpm}$ for one min. Measurements were repeated four times $(n=4)$ and the average and standard deviation $(\mathrm{SD})$ of these measurements were determined.

\subsection{Analysis of Breakage Kinetics and Energy Consumption}

A time constant was defined to characterize the breakage kinetics of the drug particles: $t d 50$ which is the time required for the drug particles to reach a medium particle size of $0.25 \mu \mathrm{m}$. The time constant was determined by pchip function in MATLAB, in which piecewise cubic Hermite polynomial interpolation was used. The specific energy consumed by the mill at this characteristic time constant was calculated as follows:

$$
E_{t d 50}=\frac{P_{w} V_{m} t_{d 50}}{m_{D}}
$$

where $V_{m}$ is the volume of the milling chamber and $m_{D}$ is the total mass of the drug in the suspension. Before the merit score calculation (Equation (3)), the values of $t_{d 50}$ and $E_{t d 50}$ were normalized based on their maximum and minimum values, as shown in Equations (2) and (3), respectively. The merit score quantifies the degree to which the milling process simultaneously achieves faster breakage and lower specific energy consumption. The higher the merit score is, the more desirable the milling process is from the perspective of cycle time reduction and minimization of energy consumption/costs.

$$
\begin{gathered}
\overline{t_{d 50}}=\frac{t_{d 50}}{t_{d 50, \text { max }}-t_{d 50, \min }} \\
\overline{E_{t d 50}}=\frac{E_{t d 50}}{E_{t d 50, \max }-E_{t d 50, \min }} \\
\text { Merit score }=\frac{2}{\overline{t_{d 50}}+\overline{E_{t d 50}}}
\end{gathered}
$$

\section{Results and Discussion}

As can be seen in Figure 2, all suspensions have been successfully milled to reach a median particle size $d_{50}$ size smaller than $0.165 \mu \mathrm{m}$. Both the median size $d_{50}$ and $90 \%$ passing particle size $d_{90}$ decreased monotonically during the milling and $d_{50}$ attained a plateau size, known as the grinding limit, whereas $d_{90}$ approached a plateau. The monotonicity of the timewise variation of $d_{50}-d_{90}$ reflects the fact that the particle breakage occurred faster and to a larger extent than the particle aggregation during the milling. Overall, these dynamic features are in agreement with our earlier work for wellstabilized drug nanosuspensions [22-25]. Particle aggregation was largely suppressed owing to the electrosteric stabilization provided by the neutral adsorbing polymer (HPC)-anionic surfactant (SDS) combination [19-21]. Since the formulation selection here was done based on earlier studies, the nanosuspension produced was physically stable, as confirmed by negligible size increase upon 7-day ageing (results not shown here for the sake of brevity).

At higher YSZ bead concentration, i.e., higher cYsz, the drug particle breakage occurred faster, leading to a smaller $d_{50}$ at a given time until a dynamic equilibrium was reached. The discrimination between the performances of the mixtures can be observed more clearly in the first minutes of the experiments. However, as particle size decreases, the impact of bead material gets less significant. When specific time constants are considered, we can clearly note a trend where the time needed for the median particle size to reach $0.25 \mu \mathrm{m} t d 50$ decreased with increasing YSZ concentration (Figure 3a). This impact got weaker at higher YSZ concentration (especially above $c$ YSZ $>0.5$ ). Parker et al. [15] used a microhydrodynamic model to explain why YSZ alone performed better than CPS alone. They found that owing to its larger density, the use of YSZ beads alone led more frequent and energetic/forceful bead-bead collisions and more frequent drug particle compressions as compared 
with the use of CPS beads alone. This finding could explain why the mixtures with higher YSZ concentration resulted in faster drug particle breakage.
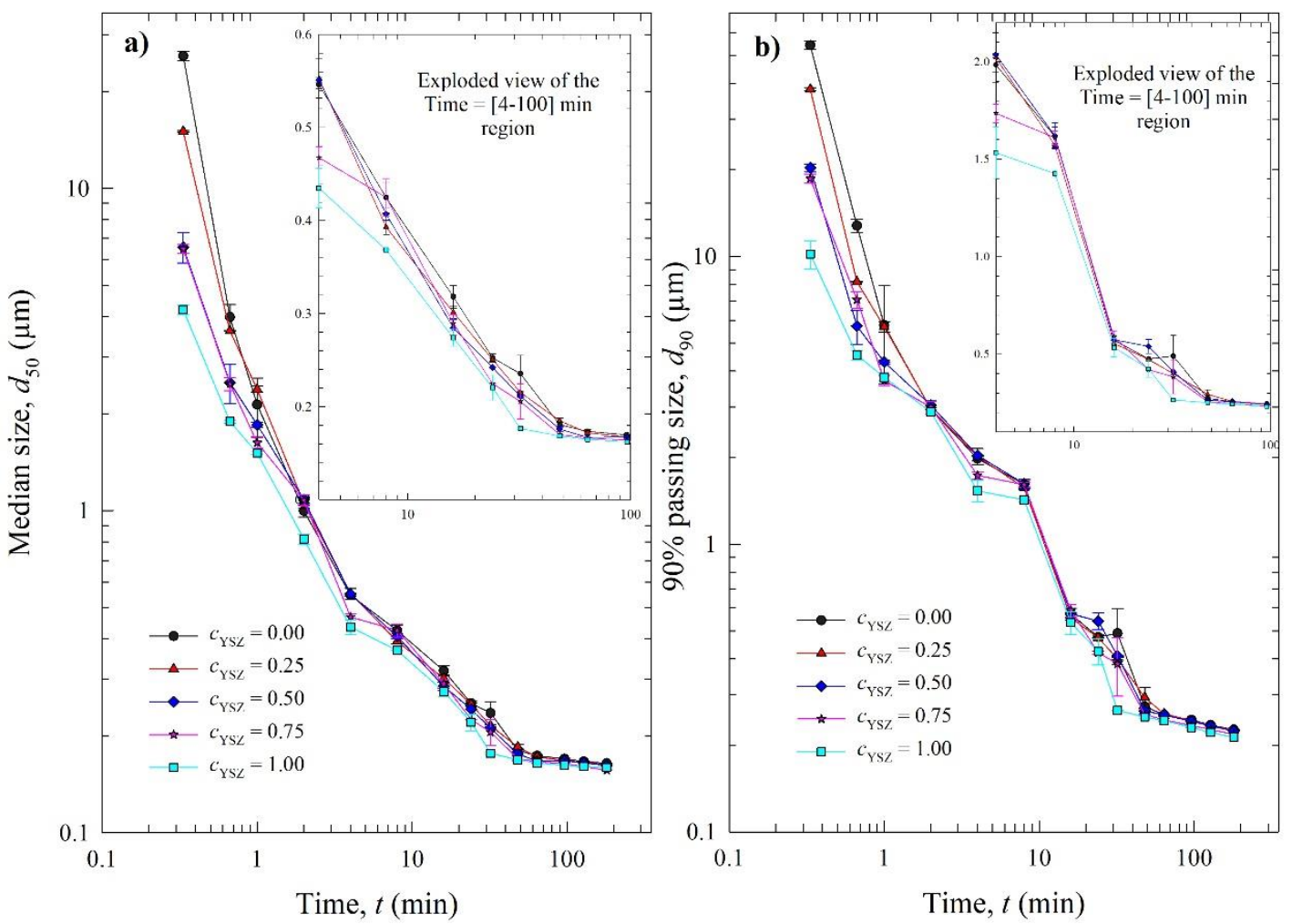

Figure 2. Effects of YSZ concentrations in the mixture on the time-wise evolution of $d_{50}$ and $d_{90}$ during the milling of FNB particles.

Upon an increase in CYSZ, the power required by the mill increased drastically (Figure 3b). This can be attributed to higher density of YSZ beads than that of CPS beads. The specific energy consumption also increased, albeit to a lesser extent than that for the stirrer power (see Figure 3c) because the higher stirrer power was applied for a shorter time to attain $0.25 \mu \mathrm{m}$ at higher $c$ Ysz. It appears that the use of YSZ beads is advantageous from a cycle time perspective as it lead to faster breakage, thus enabling reduction in manufacturing time. However, it is more costly because of the higher stirrer power required and higher specific energy consumption. The use of CPS beads require lower stirrer power and specific energy consumption. The merit score in Equation (3) considers both cycle time and specific energy consumption. Figure 3d illustrates that 25\%CPS-75\% YSZ mixture had the highest merit score (optimal process). Although the merit scores were not dramatically different among the bead mixtures, a bead mixture could make best use of the two types of beads. 

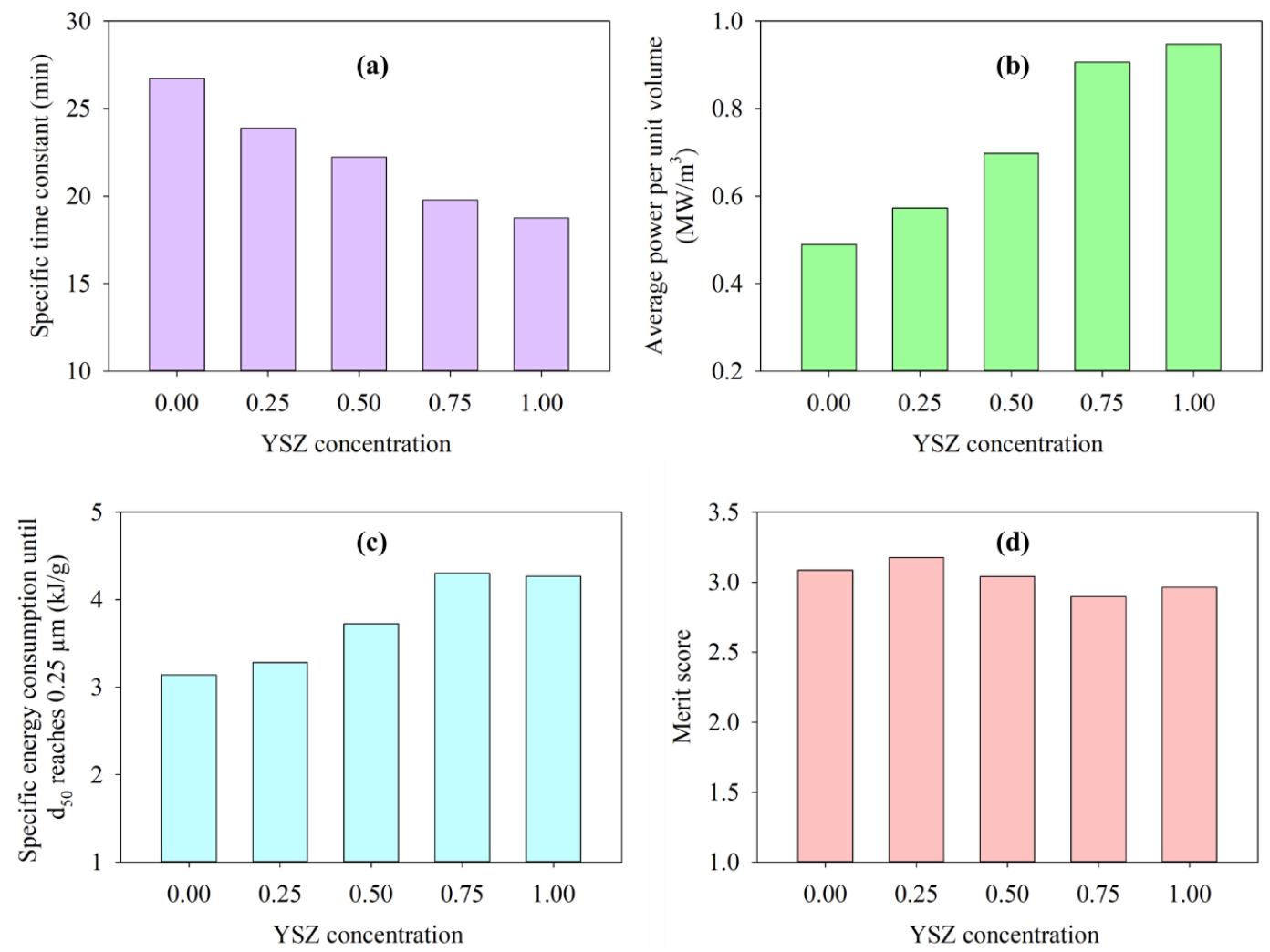

Figure 3. Effects of YSZ concentration on (a) characteristic time constant, td50, (b) Average stirrer power per unit volume $\mathrm{Pw}$, (c) specific energy consumption until the d50 reaches $0.25 \mu \mathrm{m}$, and (d) Merit score.

\section{Conclusions}

In this study, we have investigated the impact of combined use of two types of beads, CPS and YSZ, as a mixture on the breakage kinetics and energy consumption during the wet stirred media milling. For this reason, five milling experiments were conducted by using five different bead mixtures with different YSZ concentrations. While YSZ beads led to faster breakage and shorter cycle time than CPS beads, they required a higher stirrer power and specific energy consumption. However, when power consumption and breakage kinetics was considered together in view of the calculated merit scores, an optimum was identified for a 25\% CPS-75\% YSZ bead mixture. Hence, this is the first study in pharmaceutical nanotechnology literature that paves the way to process optimization via use of bead mixtures.

The merit score is an overly simplistic parameter defined to assess overall process performance considering both breakage kinetics/cycle time and energy-efficiency/utility costs of the process. Based on full operational considerations and process economics, more realistic and comprehensive merit scores can be defined for process optimization. While cycle time is of prime importance in pharmaceutical industry as compared with energy consumption/costs, the consideration of specific energy consumption in the merit score was warranted for other reasons. The processes associated with high stirrer power and specific energy consumption are likely to have other concerns such as significant heat generation and the need for excellent cooling as well as higher extent of media contamination, which have not been explicitly studies here. Therefore, the selection of bead material depends on the holistic needs of the pharmaceutical industry. Finally, a different bead mixture could be optimal based on different prioritization of the various considerations (cycle time, energy consumption, temperature control, contamination), which will be considered in future studies. 
Author Contributions: Conceptualization, E.B.; Formal analysis, G.G. and E.B.; Investigation, G.G., M.K., M.B. and N.P.; Methodology, G.G. and E.B.; Project administration, E.B.; Resources, E.B.; Supervision, G.G. and E.B.; Validation, G.G.; Writing-original draft, G.G.; Writing-review \& editing, E.B. All authors have read and agreed to the published version of the manuscript.

Acknowledgments: G.G. acknowledges the NJIT Department of Chemical and Materials Engineering for the financial support, while E.B. acknowledges the Faculty Instrument Usage Seed Grant (FIUSG) by NJIT Otto York Center \& the Materials Characterization Lab for characterization support.

Conflicts of Interest: The authors declare no conflict of interest.

\section{Abbreviations}

The following abbreviations are used in this manuscript:

$\begin{array}{ll}\text { WSMM } & \text { Wet Stirred Media Milling } \\ \text { YSZ } & \text { Ytrium stabilized zirconia beads } \\ \text { CPS } & \text { Crosslinked polystyrene beads } \\ d_{50} & \text { median particle size } \\ t_{d 50} & \text { time required for d50 to reach } 0.25 \mu \mathrm{m} \\ E_{\mathrm{td} 50} & \text { Specific energy consumption during td50 } \\ \mathrm{P}_{\mathrm{w}} & \text { Power required for stirrer during milling }\end{array}$

\section{References}

1. Lipinski, C. Poor aqueous solubility-An industry wide problem in drug discovery. Am. Pharm. Rev. 2002, $5,82-85$.

2. Kesisoglou, F.; Panmai, S.; Wu, Y. Nanosizing-Oral formulation development and biopharmaceutical evaluation. Adv. Drug Deliv. Rev. 2007, 59, 631-644.

3. Bhakay, A.; Merwade, M.; Bilgili, E.; Dave, R.N. Novel aspects of wet milling for the production of microsuspensions and nanosuspensions of poorly water-soluble drugs. Drug Dev. Ind. Pharm. 2011, 37, $963-$ 976.

4. Malamatari, M.; Taylor, K.M.G.; Malamataris, S.; Douroumis, D.; Kachrimanis, K. Pharmaceutical nanocrystals: Production by wet milling and applications. Drug Discov. Today 2018, 23, 534-547.

5. Chen, H.; Khemtong, C.; Yang, X.; Chang, X.; Gao, J. Nanonization strategies for poorly water-soluble drugs. Drug Discov. Today 2011, 16, 354-360.

6. Mohammad, I.S.; Hu, H.; Yin, L.; He, W. Drug nanocrystals: Fabrication methods and promising therapeutic applications. Int. J. Pharm. 2019, 562, 187-202.

7. Bhakay, A.; Rahman, M.; Dave, R.N.; Bilgili, E. Bioavailability Enhancement of Poorly Water-Soluble Drugs via Nanocomposites: Formulation-Processing Aspects and Challenges. Pharmaceutics 2018, 10, 86.

8. Li, M.; Azad, M.; Davé, R.; Bilgili, E. Nanomilling of Drugs for Bioavailability Enhancement: A Holistic Formulation-Process Perspective. Pharmaceutics 2016, 8, 17.

9. Peltonen, L. Design Space and QbD Approach for Production of Drug Nanocrystals by Wet Media Milling Techniques. Pharmaceutics 2018, 10, 104.

10. Li, M.; Yaragudi, N.; Afolabi, A.; Dave, R.; Bilgili, E. Sub-100nm drug particle suspensions prepared via wet milling with low bead contamination through novel process intensification. Chem. Eng. Sci. 2015, 130, 207-220.

11. Kawatra, S.K. Advances in Comminution; Society for Mining, Metallurgy, and Exploration: Littleton, Colorado, 2006.

12. Afolabi, A.; Akinlabi, O.; Bilgili, E. Impact of process parameters on the breakage kinetics of poorly watersoluble drugs during wet stirred media milling: A microhydrodynamic view. Eur. J. Pharm. Sci. 2014, 51, 75-86.

13. Bilgili, E.; Capece, M.; Afolabi, A. Modeling of milling processes via DEM, PBM, and microhydrodynamics. In Predictive Modeling of Pharmaceutical Unit Operations, Pandey, P., Bharadwaj, R., Eds.; Woodhead Publishing: Cambridge, UK, 2017; pp. 159-203.

14. Li, M.; Alvarez, P.; Bilgili, E. A microhydrodynamic rationale for selection of bead size in preparation of drug nanosuspensions via wet stirred media milling. Int. J. Pharm. 2017, 524, 178-192. 
15. Parker, N.; Rahman, M.; Bilgili, E. Impact of media material and process parameters on breakage kineticsenergy consumption during wet media milling of drugs. Eur. J. Pharm. Biopharm. 2020, 153, 52-67.

16. Rahman, M.; Arevalo, F.; Coelho, A.; Bilgili, E. Hybrid nanocrystal-amorphous solid dispersions (HyNASDs) as alternative to ASDs for enhanced release of BCS Class II drugs. Eur. J. Pharm. Biopharm. 2019, 145, 12-26.

17. Rahman, M.; Ahmad, S.; Tarabokija, J.; Bilgili, E. Roles of surfactant and polymer in drug release from spray-dried hybrid nanocrystal-amorphous solid dispersions (HyNASDs). Powder Technol. 2020, 361, 663678.

18. Jamzad, S.; Fassihi, R. Role of surfactant and $\mathrm{pH}$ on dissolution properties of fenofibrate and glipizide $-\mathrm{A}$ technical note. Aaps Pharmscitech 2006, 7, 17-22.

19. Azad, M.; Afolabi, A.; Bhakay, A.; Leonardi, J.; Davé, R.; Bilgili, E. Enhanced physical stabilization of fenofibrate nanosuspensions via wet co-milling with a superdisintegrant and an adsorbing polymer. Eur. J. Pharm. Biopharm. 2015, 94, 372-385.

20. Bilgili, E.; Li, M.; Afolabi, A. Is the combination of cellulosic polymers and anionic surfactants a good strategy for ensuring physical stability of BCS Class II drug nanosuspensions? Pharm. Dev. Technol. 2016, 21, 499-510.

21. Knieke, C.; Azad, M.A.; Davé, R.N.; Bilgili, E. A study of the physical stability of wet media-milled fenofibrate suspensions using dynamic equilibrium curves. Chem. Eng. Res. Des. 2013, 91, 1245-1258.

22. Monteiro, A.; Afolabi, A.; Bilgili, E. Continuous production of drug nanoparticle suspensions via wet stirred media milling: A fresh look at the Rehbinder effect. Drug Dev. Ind. Pharm. 2013, 39, 266-283.

23. Bilgili, E.; Afolabi, A. A combined microhydrodynamics-polymer adsorption analysis for elucidation of the roles of stabilizers in wet stirred media milling. Int. J. Pharm. 2012, 439, 193-206.

24. Bilgili, E.; Rahman, M.; Palacios, D.; Arevalo, F. Impact of polymers on the aggregation of wet-milled itraconazole particles and their dissolution from spray-dried nanocomposites. Adv. Powder Technol. 2018, 29, 2941-2956.

25. Li, M.; Alvarez, P.; Orbe, P.; Bilgili, E. Multi-faceted Characterization of Wet-milled Griseofulvin Nanosuspensions for Elucidation of Aggregation State and Stabilization Mechanisms. Aaps Pharmscitech 2018, 19, 1789-1801.

Publisher's Note: MDPI stays neutral with regard to jurisdictional claims in published maps and institutional affiliations.

(C) 2020 by the authors. Submitted for possible open access publication under the terms and conditions of the Creative Commons Attribution (CC BY) license (http://creativecommons.org/licenses/by/4.0/). 